\title{
Tratamento da estomatite aftosa recorrente: uma revisão integrativa da literatura
}

\section{Treatment of recurrent aphthous stomatitis: an integrative literature review}

\author{
Anna Clara Fontes Vieira* \\ Cadidja Dayane Sousa do Carmo* \\ Gleusa Mara Barreto Vieira* \\ Leonardo Abrantes Lima** \\ Maria Carmen Fontoura Nogueira da Cruz ${ }^{* * *}$ \\ Fernanda Ferreira Lopes ${ }^{* * *}$
}

\section{Resumo}

Objetivo: realizar uma revisão integrativa de publicações científicas sobre os tratamentos da estomatite aftosa recorrente, na busca de subsídios para guiar a prática clínica no manejo dessa condição. Revisão de literatura: a estomatite aftosa recorrente é caracterizada pelo aparecimento de úlceras na mucosa bucal, apresentando-se de forma dolorosa e levando a um impacto negativo na qualidade de vida dos pacientes. Este trabalho é resultado de uma pesquisa sobre a estomatite aftosa recorrente nas seguintes bases de dados: PubMed (National Center for Biotechnology Information and National Library of Medicine), The Cochrane Library, Scopus (The Scopus Cited Reference Expansion), utilizando-se os seguintes descritores: recurring aphthous stomatitis or recurrent aphthous stomatitis or recurrent aphthous ulcer or recurrent aphthous ulceration and treatment. Foram selecionados 23 estudos de acordo com os critérios de inclusão e exclusão adotados. Esses estudos identificaram a utilização de diversos medicamentos, que variam de medicamentos naturais a laser, tanto com uso tópico como sistêmico, com resultados satisfatórios para o manejo da estomatite aftosa recorrente. Considerações finais: o presente estudo considerou as terapias tópicas, como um todo, uma opção de tratamento eficaz e com menores possibilidades de efeitos colaterais. Em relação às terapias sistêmicas, os resultados permanecem inconclusivos.

Palavras-chave: Estomatite aftosa. Tratamento. Patologia.

\section{Introdução}

A estomatite aftosa recorrente (EAR) é uma condição comum caracterizada por úlceras múltiplas, pequenas, ovoides, com margem circunscrita, halo eritematoso e fundo amarelo ou cinza, com aparecimento típico primeiramente na infância ou adolescência ${ }^{1}$. A manifestação dessa alteração dá-se em surtos e com um curso crônico, com tempo limitado da lesão, na maior parte dos $\operatorname{casos}^{2,3}$.

Do ponto de vista epidemiológico, a EAR é uma das mais comuns desordens orais, conhecida por afetar cerca de $20 \%$ da população em algum momento da vida 4 . Apesar de sua alta prevalência na população, sua etiopatogenia perdura como um enigma, pois, mesmo com muitas investigações, os estudos têm falhado em encontrar a sua exata etio$\operatorname{logia}^{5}$. Entretanto, diversos fatores de ordem local, sistêmico, imunológico, genético, alérgico, nutricional e microbiano têm sido propostos como possíveis agentes causadores ${ }^{3}$.

Existem diversas linhas de evidências indicando que leucócitos polimorfonucleares (PMN) têm um papel importante na patogênese da EAR. Alguns mediadores inflamatórios, como o fator de necrose tumoral alfa (TNF- $\alpha$ ), lipopolissacarídeos e macrófagos/granulócitos podem desencadear a ativação funcional de PMN. Desse modo, a ativação excessiva

Doutorandas em Odontologia, Universidade Federal do Maranhão, São Luís, MA, Brasil.

Mestrando(a) em Odontologia, Universidade Federal do Maranhão, São Luís, MA, Brasil.

*** Doutoras em Patologia Oral, Universidade Federal do Rio Grande do Norte, professoras adjuntas, Curso de Odontologia, Universidade Federal do Maranhão, São Luís, MA, Brasil. 
e desnecessária, o prolongamento do tempo de vida de um PMN ou o aumento da sua capacidade bactericida podem produzir substâncias tóxicas para 0 tecido sadio ${ }^{6-8}$. Adicionalmente, estudos patológicos de úlceras aftosas têm revelado que o TNF- $\alpha$ está presente principalmente em macrófagos/monócitos e linfócitos contidos no infiltrado inflamatório mononuclear que está presente nessas lesões ${ }^{9}$.

Três subtipos clínicos da EAR têm sido estabelecidos de acordo com seu tamanho, número, duração e agudizações: EAR menor, EAR maior, EAR herpetiforme. $\mathrm{O}$ primeiro é a apresentação mais comum da doença, representando de $70 \%$ a $85 \%$ de todos os casos, envolve úlceras com menos de $1 \mathrm{~cm}$ e duração de quatro a quatorze dias, em média. A EAR maior representa $10 \%$ dos casos, atingindo mais de $1 \mathrm{~cm}$ de diâmetro e perdurando em torno de seis semanas. Já a herpetiforme atinge uma média de $1 \%$ a $10 \%$ dos casos, sendo profundas e com média de 2 $\mathrm{cm}$ a $3 \mathrm{~cm}$, com possibilidade de aparecimento de mais de cem aftas simultâneas ${ }^{10}$.

Essas úlceras apresentam-se de forma dolorosa, levando a um impacto negativo na qualidade de vida dos pacientes ${ }^{11}$, podendo restringir a ingestão de alguns alimentos e também comprometer uma adequada higiene oral, possibilitando um maior acúmulo de biofilme oral, o que aumenta o risco para o desenvolvimento de outros agravos bucais, como a doença periodontal e a cárie dentária ${ }^{12}$. Dessa forma, a EAR representa um problema que requer atenção, devido à sua alta prevalência e associação com dor e influência na qualidade de vida dos indivíduos afetados ${ }^{13}$.

Como tratamento, são indicados variados agentes terapêuticos, dentre eles, medicamentos sistêmicos e agentes tópicos têm sido utilizados na redução dos sintomas e na busca de uma acelerada regeneração dessa lesão ${ }^{14,15}$, tais como a utilização de produtos naturais ${ }^{16}$, antibióticos ${ }^{5}$, anti-inflamatórios ${ }^{17}$, vitaminas ${ }^{18}$ ou laser $^{19}$.

Desse modo, tendo em vista a alta prevalência da estomatite aftosa recorrente, suas implicações na qualidade de vida do paciente e suas inúmeras alternativas de tratamento, o presente trabalho busca realizar uma revisão integrativa de publicações científicas sobre os tratamentos da estomatite aftosa recorrente, no intuito de subsidiar a prática clínica no manejo dessa condição.

\section{Metodologia}

O presente estudo é caracterizado como uma revisão integrativa da literatura. Esse tipo de trabalho consiste na análise crítica de artigos científicos relevantes que possibilitam a síntese do estado do conhecimento de um determinado assunto, dando suporte para a tomada de decisão e melhoria da prática clíni$\mathrm{ca}^{20}$. O tema escolhido para essa revisão integrativa da literatura foi tratamento da úlcera aftosa recorrente, gerando a seguinte pergunta de pesquisa: Qual tratamento indicar para úlcera aftosa recorrente?
Os critérios de inclusão adotados na seleção dos artigos foram: tipo de estudo (ensaios clínicos fases I, II, III e IV, ensaios clínicos controlados, ensaios clínicos controlados randomizados, metanálises, revisões sistemáticas da literatura, e estudos observacionais do tipo coorte), sujeitos da pesquisa (seres humanos), idioma de publicação (língua inglesa) e ano de publicação (2010 a 2014). Foram excluídos da pesquisa aqueles artigos não disponíveis de forma integral pelo acesso do portal de periódicos da Coordenação de Aperfeiçoamento de Pessoal de Nível Superior (Capes).

Os descritores foram selecionados e combinados da seguinte forma: recurring aphthous stomatitis or recurrent aphthous stomatitis or recurrent aphthous ulcer OR recurrent aphthous ulceration and treatment. A pesquisa por trabalhos científicos foi realizada na sessão de busca avançada das seguintes bases de dados: PubMed (National Center for Biotechnology Information and National Library of Medicine), The Cochrane Library e Scopus (The Scopus Cited Reference Expansion).

Com a utilização dos filtros, de acordo com os critérios de inclusão pré-estabelecidos, a base PubMed retornou cinquenta títulos, a base The Cochrane Library retornou dois, e a base Scopus oitenta títulos. A partir dessa busca, os artigos foram analisados quanto à repetição, quando se detectou três títulos repetidos. Em seguida, os trabalhos foram selecionados, primeiramente pelo título, por dois examinadores independentes, confrontando-se as duas seleções para a definição dos títulos que seriam definitivamente incluídos. Após essa etapa, houve a seleção dos artigos pelo resumo, seguindo os mesmos critérios utilizados para a seleção dos títulos (dois examinadores independentes e confronto das duas seleções), e finalmente, após a seleção pelo resumo, os trabalhos foram lidos integralmente e definiu-se a amostra final do estudo, totalizando 23 artigos (Figura 1).

Foi elaborado um instrumento para a sumarização dos trabalhos, que incluiu: título do trabalho, ano de publicação, base de dados em que foi encontrado, autoria, objetivo e principais resultados e conclusões. Em seguida, procedeu-se à análise dos resultados e discussão.

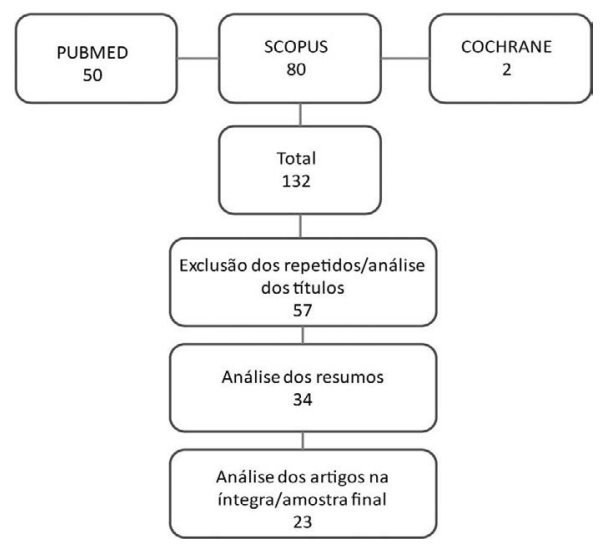

Figura 1 - Fluxograma do processo de seleção dos artigos Fonte: elaboração dos autores com base nos dados da pesquisa. 


\section{Resultados}

Nesta revisão integrativa da literatura, foram analisados 23 artigos publicados nos últimos cinco anos (2010 a 2014) acerca do tratamento da estomatite aftosa recorrente. Os artigos analisados que preencheram os critérios de inclusão previamente estabelecidos estão sumarizados nos Quadros 1, 2 e 3 , segundo o título do trabalho, o ano de publicação, a autoria, o tipo de estudo, o objetivo, os principais resultados e conclusões.

Quadro 1 - Síntese dos estudos incluídos na amostra que utilizaram produtos naturais para o tratamento da EAR segundo título, ano, autoria tipo de estudo, objetivos, resultados e conclusões

\begin{tabular}{|c|c|c|c|}
\hline Título & Autoria & \multirow{2}{*}{ Objetivos } & \multirow{2}{*}{ Resultados/conclusões } \\
\hline Ano & Tipo de estudo & & \\
\hline $\begin{array}{l}\text { Effects of camel thorn } \\
\text { distillate on recurrent oral } \\
\text { aphthous lesions }\end{array}$ & Pourahmad et al. ${ }^{21}$ & \multirow{2}{*}{$\begin{array}{l}\text { Avaliar o efeito do destilado } \\
\text { da leguminosa espinho de } \\
\text { camelo (Alhagi camelorum) } \\
\text { na EAR (uso tópico). }\end{array}$} & \multirow{2}{*}{$\begin{array}{l}\text { Após o quinto dia de tratamento a escala de } \\
\text { dor e o diâmetro da lesão alcançaram nível } \\
\text { zero no grupo de estudo, enquanto que no } \\
\text { grupo controle não houve redução total até o } \\
14^{\circ} \text { dia. Dessa forma, o destilado de espinho } \\
\text { de camelo tem sua eficácia comparada a } \\
\text { outras drogas utilizadas para tratar EAR. }\end{array}$} \\
\hline 2010 & $\begin{array}{l}\text { Ensaio clínico controla- } \\
\text { do randomizado }\end{array}$ & & \\
\hline $\begin{array}{l}\text { Management of aphthous } \\
\text { ulceration with topical } \\
\text { quercetin: a randomized } \\
\text { clinical trial }\end{array}$ & Hamdy e Ibrahem ${ }^{22}$ & \multirow[b]{2}{*}{$\begin{array}{l}\text { Determinar o efeito da apli- } \\
\text { cação tópica de quercetina } \\
\text { no tratamento de úlceras af- } \\
\text { tosas menores. }\end{array}$} & \multirow{2}{*}{$\begin{array}{l}\text { A aplicação tópica de creme de quercetina } \\
\text { para aftas menores aliviou a dor e produziu } \\
\text { a cura completa em } 35 \% \text { dos pacientes, } \\
\text { em dois a quatro dias, alcançando a cura } \\
\text { em } 100 \% \text { dos pacientes em sete a dez dias } \\
\text { de tratamento. Apresentou ainda maior } \\
\text { redução do tamanho da lesão depois de } \\
\text { dez dias em relação ao grupo controle ( } p \\
<0,004) \text {. A quercetina é um promissor trata- } \\
\text { mento novo, adjuvante seguro, bem tolera- } \\
\text { do e altamente eficaz para a cura de úlceras } \\
\text { aftosas comuns. }\end{array}$} \\
\hline 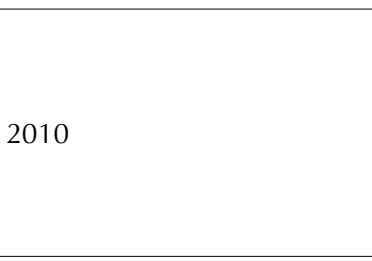 & $\begin{array}{l}\text { Ensaio clínico randomi- } \\
\text { zado controlado }\end{array}$ & & \\
\hline $\begin{array}{l}\text { The efficacy of a paste } \\
\text { containing Myrtuscommunis } \\
\text { (Myrtle) in the management } \\
\text { of recurrent aphthous } \\
\text { stomatitis: a randomized } \\
\text { controlled trial }\end{array}$ & Babaee et al. ${ }^{23}$ & \multirow{2}{*}{$\begin{array}{l}\text { Verificar a eficácia clínica e } \\
\text { segurança de uma pasta oral } \\
\text { à base de mirta comum (apli- } \\
\text { cação tópica) no tratamento } \\
\text { da estomatite aftosa recorren- } \\
\text { te (EAR). }\end{array}$} & \multirow{2}{*}{$\begin{array}{l}\text { Houve diferença significante }(p<0,001) \text { em } \\
\text { todos os critérios para avaliação da eficácia } \\
\text { da pasta entre o grupo controle e o teste: } \\
\text { diâmetro, dor, OHIP }-14 \text { (perfil do impacto } \\
\text { de saúde bucal), exsudato, severidade. A } \\
\text { pasta à base de mirta comum a } 5 \% \text { foi bem } \\
\text { tolerada e efetiva no tratamento da EAR. Po- } \\
\text { rém, estudos com maior número de sujei- } \\
\text { tos, visando à prevenção quando no estágio } \\
\text { prodrômico, são necessários. }\end{array}$} \\
\hline 2010 & $\begin{array}{l}\text { Ensaio clínico randomi- } \\
\text { zado controlado }\end{array}$ & & \\
\hline $\begin{array}{l}\text { Clinical evaluation of allicin } \\
\text { oral adhesive tablets in } \\
\text { the treatment of recurrent } \\
\text { aphthous ulceration }\end{array}$ & Jiang et al. ${ }^{24}$ & \multirow{2}{*}{$\begin{array}{l}\text { Investigar a eficácia e a segu- } \\
\text { rança da aplicação tópica de } \\
5 \text { mg de alicina (adesivos) no } \\
\text { tratamento de pequenas ul- } \\
\text { cerações aftosas recorrentes. }\end{array}$} & \multirow{2}{*}{$\begin{array}{l}\text { Houve redução significativa no tamanho da } \\
\text { úlcera e no alívio da dor em } 2,4 \text { e } 6 \text { dias } \\
\text { de tratamento quando comparados com o } \\
\text { placebo. }\end{array}$} \\
\hline 2012 & $\begin{array}{l}\text { Ensaio clínico randomi- } \\
\text { zado controlado }\end{array}$ & & \\
\hline $\begin{array}{l}\text { Effects of berberine gelatin } \\
\text { on recurrent aphthous } \\
\text { stomatitis: a randomized, } \\
\text { placebo-controlled, double- } \\
\text { blind trial in a Chinese } \\
\text { cohort }\end{array}$ & Jiang et al. ${ }^{25}$ & \multirow{2}{*}{$\begin{array}{l}\text { Investigar a eficácia e segu- } \\
\text { rança da gelatina de berberi- } \\
\text { na (aplicação tópica) no tra- } \\
\text { tamento da estomatite aftosa } \\
\text { recorrente menor. }\end{array}$} & \multirow{2}{*}{$\begin{array}{l}\text { O uso da gelatina de berberina ( } 50 \mathrm{mg} \text { ) foi } \\
\text { eficaz na redução da dor, diminuição do } \\
\text { tamanho da úlcera, diminuição do eritema } \\
\text { e exsudato. Nenhum efeito colateral foi ob- } \\
\text { servado pelo uso da gelatina de berberina. }\end{array}$} \\
\hline 2013 & $\begin{array}{l}\text { Ensaio clínico rando- } \\
\text { mizado, duplo cego e } \\
\text { controlado por placebo }\end{array}$ & & \\
\hline $\begin{array}{l}\text { The efficacy of Kamistad } \\
\text { gel in the management of } \\
\text { recurrent minor aphthous } \\
\text { ulceration }\end{array}$ & He et al. ${ }^{26}$ & \multirow{2}{*}{$\begin{array}{l}\text { Avaliar a eficácia e a segu- } \\
\text { rança do gel de Kamistad no } \\
\text { tratamento da úlcera aftosa } \\
\text { recorrente, particularmente, } \\
\text { quando aplicado (tópico) no } \\
\text { início dos sintomas. }\end{array}$} & \multirow{2}{*}{$\begin{array}{l}\text { O grupo prodrômico apresentou redução } \\
\text { do tamanho e duração da úlcera }(p<0,01) \text {, } \\
\text { em relação ao grupo com úlcera instalada, } \\
\text { não havendo diferença em relação à dor } \\
(p=0,236) \text {. No tratamento da ulceração af- } \\
\text { tosa recorrente, o uso do gel de Kamistad no } \\
\text { início dos sintomas prodrômicos pode preve- } \\
\text { nir a progressão do desenvolvimento da úl- } \\
\text { cera e reduzir significantemente os sintomas } \\
\text { caso a úlcera venha a se desenvolver. }\end{array}$} \\
\hline 2014 & $\begin{array}{l}\text { Ensaio clínico randomi- } \\
\text { zado cego }\end{array}$ & & \\
\hline $\begin{array}{l}\text { The effect of collagenic gels } \\
\text { with marine algae extracts } \\
\text { mixtures in the treatment of } \\
\text { recurrent aphthous stomatitis }\end{array}$ & Bechir et al. ${ }^{27}$ & \multirow{2}{*}{$\begin{array}{l}\text { Determinar a tolerabilidade } \\
\text { e o processo de cicatrização } \\
\text { de seis produtos feitos à base } \\
\text { de extrato de algas marinhas } \\
\text { combinados com gel de co- } \\
\text { lágeno de couro de peixes } \\
\text { (tubarão) no tratamento tópi- } \\
\text { co da EAR. }\end{array}$} & \multirow{2}{*}{$\begin{array}{l}\text { Maior efetividade foi apresentada pelo gel à } \\
\text { base de Ceraminum rubrum a } 10 \% \text {. Haven- } \\
\text { do sucesso do tratamento dos géis utilizados } \\
\text { em relação à redução da dor e do tamanho } \\
\text { das lesões decorrente da EAR, com destaque } \\
\text { para o Ceraminum rubrum a } 10 \% \text {. }\end{array}$} \\
\hline 2014 & Ensaio clínico & & \\
\hline
\end{tabular}

Fonte: todos os quadros são de elaboração dos autores com base nos dados da pesquisa. 
Quadro 2 - Síntese dos estudos incluídos na amostra que utilizaram substâncias convencionais para o tratamento da EAR segundo título, ano, autoria, tipo de estudo, objetivos, resultados e conclusões

\begin{tabular}{|c|c|c|c|}
\hline Título & Autoria & \multirow{2}{*}{ Objetivos } & \multirow{2}{*}{ Resultados/conclusões } \\
\hline Ano & Tipo de estudo & & \\
\hline $\begin{array}{l}\text { The effect of ascorbate on } \\
\text { minor recurrent aphthous } \\
\text { stomatitis }\end{array}$ & $\begin{array}{l}\text { Ensaio clínico contro- } \\
\text { lado cruzado }\end{array}$ & $\begin{array}{l}\text { Investigar o potencial do } \\
\text { tratamento para prevenção } \\
\text { da EAR menor com uso de } \\
\text { ascorbato (uso sistêmico). }\end{array}$ & $\begin{array}{l}\text { A frequência de EAR menor três meses } \\
\text { antes, durante e após tratamento foi } \\
\text { de } 4.25 \pm 2 ; 0.63 \pm 0.78 ; 1.94 \pm 1.16 \text {. } \\
\text { Quanto ao nível de dor pré, durante e } \\
\text { pós-tratamento, o ascorbato parece ser } \\
\text { efetivo, mas os dados desse estudo fo- } \\
\text { ram preliminares e precisam ser avalia- } \\
\text { dos em um estudo controlado randomi- } \\
\text { zado com maior quantidade de sujeitos } \\
\text { da pesquisa. }\end{array}$ \\
\hline $\begin{array}{l}\text { Evaluation of penicillin } \\
G \text { potassium troches in } \\
\text { the treatment of minor } \\
\text { recurrent aphthous ulceration } \\
\text { in a Chinese cohort: a } \\
\text { randomized, double-blinded, } \\
\text { placebo and no-treatment- } \\
\text { controlled, multicenter } \\
\text { clinical trial }\end{array}$ & $\begin{array}{l}\text { Ensaio clínico rando- } \\
\text { mizado controlado du- } \\
\text { plo cego }\end{array}$ & $\begin{array}{l}\text { Explorar a eficácia e a segu- } \\
\text { rança da aplicação tópica } \\
\text { de } 50 \mathrm{mg} \text { de pastilhas de } \\
\text { penicilina G potássica no } \\
\text { tratamento de pequenas ul- } \\
\text { cerações aftosas recorrentes } \\
\text { em uma coorte chinesa. }\end{array}$ & $\begin{array}{l}\text { O grupo que utilizou a penicilina G po- } \\
\text { tássica apresentou redução no tamanho } \\
\text { da úlcera e na dor. Portanto, a penicili- } \\
\text { na G potássica é eficaz na redução do } \\
\text { tamanho da úlcera e alívio da dor, com } \\
\text { apresentação de poucos efeitos adver- } \\
\text { sos. }\end{array}$ \\
\hline $\begin{array}{l}\text { Comparison of colchicine } \\
\text { versus prednisolone in } \\
\text { recurrent aphthous stomatitis: } \\
\text { double-blind randomized } \\
\text { clinical trial }\end{array}$ & $\begin{array}{l}\text { Ensaio clínico rando- } \\
\text { mizado duplo cego }\end{array}$ & $\begin{array}{l}\text { Comparar os efeitos tera- } \\
\text { pêuticos de } 5 \mathrm{mg} \text { de pred- } \\
\text { nisolona com } 0,5 \mathrm{mg} \text { de } \\
\text { colchicina no tratamento da } \\
\text { EAR (uso sistêmico). }\end{array}$ & $\begin{array}{l}\text { Não houve diferença significativa no ta- } \\
\text { manho e número de lesões, recorrência } \\
\text { e gravidade da dor entre os dois gru- } \\
\text { pos. A colchicina }(52,9 \%) \text { apresentou } \\
\text { um número significativamente maior de } \\
\text { efeitos secundários do que a predniso- } \\
\text { lona ( } 11,8 \%) \text {. Desse modo, as duas te- } \\
\text { rapias tiveram uma eficácia semelhante, } \\
\text { com a apresentação de efeitos colaterais } \\
\text { mais frequente com o uso de colchicina. } \\
\text { A prednisolona parece ser uma alternati- } \\
\text { va melhor na redução dos sinais e sinto- } \\
\text { mas da doença. }\end{array}$ \\
\hline $\begin{array}{l}\text { Systemic interventions for } \\
\text { recurrent aphthous stomatitis } \\
\text { (mouth ulcers) }\end{array}$ & Brocklehurst et al. ${ }^{30}$ & $\begin{array}{l}\text { Determinar o efeito clínico } \\
\text { de intervenções sistêmicas } \\
\text { na redução da dor associa- } \\
\text { da a úlcera aftosa recorrente } \\
\text { em episódios duradouros ou } \\
\text { frequentes. }\end{array}$ & $\begin{array}{l}\text { Não houve provas suficientes para } \\
\text { apoiar ou refutar o uso de qualquer in- } \\
\text { tervenção. Nenhum tratamento apresen- } \\
\text { tou-se eficaz e, portanto, os resultados } \\
\text { ainda não são conclusivos no que diz } \\
\text { respeito à melhor intervenção sistêmi- } \\
\text { ca para a úlcera aftosa recorrente. Além } \\
\text { disso, reconhece-se que as intervenções } \\
\text { sistêmicas são reservadas para aqueles } \\
\text { pacientes que não responderam aos tra- } \\
\text { tamentos tópicos e, portanto, pode re- } \\
\text { presentar um grupo seleto de pacientes. }\end{array}$ \\
\hline $\begin{array}{l}\text { Efficacy and safety of } \\
\text { dexamethasone ointment on } \\
\text { recurrent aphthous ulceration }\end{array}$ & Liu et al. ${ }^{17}$ & \multirow{2}{*}{$\begin{array}{l}\text { Determinar a eficácia e a } \\
\text { segurança de uma pomada } \\
\text { de dexametasona (aplica- } \\
\text { ção tópica) no tratamento } \\
\text { da úlcera aftosa recorrente } \\
\text { e detectar as concentrações } \\
\text { séricas de dexametasona } \\
\text { nos pacientes. }\end{array}$} & \multirow{2}{*}{$\begin{array}{l}\text { A proporção de cura foi de } 83,33 \% \text { no } \\
\text { grupo de tratamento e } 54,70 \% \text { no grupo } \\
\text { controle }(p=0,000) \text {. Não foram obser- } \\
\text { vadas reações adversas graves. Não foi } \\
\text { detectada a presença de dexametasona } \\
\text { no soro sanguíneo dos pacientes antes } \\
\text { ou após a utilização do agente medica- } \\
\text { mentoso }(<0,502 \mathrm{mg} / \mathrm{mL} \text { ). A pomada de } \\
\text { dexametasona foi eficiente e segura no } \\
\text { tratamento de úlceras aftosas recorrentes. }\end{array}$} \\
\hline 2012 & $\begin{array}{l}\text { Ensaio clínico rando- } \\
\text { mizado controlado }\end{array}$ & & \\
\hline $\begin{array}{l}\text { Multivitamin therapy for } \\
\text { recurrent aphthous stomatitis: } \\
\text { a randomized, double- } \\
\text { masked, placebo-controlled } \\
\text { trial }\end{array}$ & Lalla et al. ${ }^{31}$ & \multirow{2}{*}{$\begin{array}{l}\text { Avaliar o efeito de suplemen- } \\
\text { tação polivitamínica (sistê- } \\
\text { mica) diária na quantidade e } \\
\text { na duração dos episódios de } \\
\text { úlcera aftosa recorrente. }\end{array}$} & \multirow{2}{*}{$\begin{array}{l}\text { Os resultados não mostraram diferença } \\
\text { significativa no número médio de novos } \\
\text { episódios entre a suplementação polivi- } \\
\text { tamínica e o uso de placebo durante o } \\
\text { estudo }(p=0,69) \text {. A duração média dos } \\
\text { novos episódios de úlcera aftosa recor- } \\
\text { rente também foi semelhante para o uso } \\
\text { de vitaminas e de placebo ( } p=0,60) \text {. } \\
\text { Além disso, os autores não encontraram } \\
\text { diferenças em relação à dor, normali- } \\
\text { dade da dieta ou conformidade com o } \\
\text { regime de medicação do estudo. }\end{array}$} \\
\hline 2012 & $\begin{array}{l}\text { Ensaio clínico rando- } \\
\text { mizado controlado }\end{array}$ & & \\
\hline
\end{tabular}


cont.

\begin{tabular}{|c|c|c|c|}
\hline $\begin{array}{l}\text { Double-blind, randomized, } \\
\text { controlled clinical trial of } \\
\text { the effects of diosmectite } \\
\text { and basic fibroblast growth } \\
\text { factor paste on the treatment } \\
\text { of minor recurrent aphthous } \\
\text { stomatitis }\end{array}$ & Jiang et al. ${ }^{16}$ & \multirow{2}{*}{$\begin{array}{l}\text { Avaliar a efetividade e a se- } \\
\text { gurança da aplicação tópica } \\
\text { de diosmectite (DS) e pasta } \\
\text { de fator de crescimento de } \\
\text { fibroblasto básico (bFGF) no } \\
\text { tratamento de úlcera aftosa } \\
\text { recorrente. }\end{array}$} & \multirow{2}{*}{$\begin{array}{l}\text { A pasta de DS + bFGF reduziu signifi- } \\
\text { cativamente os escores de dor ( } p<0,05 \\
\text { para os dias } 3,4,5 \text { e } 6 \text { ), em compara- } \\
\text { ção com as outras pastas. O tamanho da } \\
\text { úlcera foi significativamente reduzido } \\
\text { ( } p<0,05 \text { para os dias } 2,4 \text { e } 6 \text { ) no grupo } \\
\text { DS + bFGF em comparação com os ou- } \\
\text { tros grupos. Não foram observados efei- } \\
\text { tos adversos evidentes da droga. Pasta } \\
\text { contendo DS + bFGF foi eficaz no alívio } \\
\text { da dor da úlcera e promoveu a cura sem } \\
\text { efeitos colaterais significativos no trata- } \\
\text { mento de estomatites recorrentes. }\end{array}$} \\
\hline 2013 & $\begin{array}{l}\text { Ensaio clínico rando- } \\
\text { mizado controlado }\end{array}$ & & \\
\hline $\begin{array}{l}\text { Single application of topical } \\
\text { doxycycline hyclate in the } \\
\text { management of recurrent } \\
\text { aphthous stomatitis }\end{array}$ & Vijayabala et al..$^{5}$ & \multirow{2}{*}{$\begin{array}{l}\text { Avaliar a eficácia da aplica- } \\
\text { ção tópica única de doxici- } \\
\text { clina como regime terapêu- } \\
\text { tico na redução da dor em } \\
\text { úlceras aftosas recorrentes. }\end{array}$} & \multirow{2}{*}{$\begin{array}{l}\text { Pacientes tratados com doxiciclina apre- } \\
\text { sentaram menor dor }(p<0,01) \text { e cura } \\
\text { mais rápida }(p<0,01) \text { quando compara- } \\
\text { dos com placebo. Uma única aplicação } \\
\text { de doxiciclina causa diminuição da dor } \\
\text { e recuperação acelerada. }\end{array}$} \\
\hline 2013 & $\begin{array}{l}\text { Ensaio clínico rando- } \\
\text { mizado controlado }\end{array}$ & & \\
\hline $\begin{array}{l}\text { Novel material in the } \\
\text { treatment of minor oral } \\
\text { recurrent aphthous stomatitis }\end{array}$ & Halim et al. ${ }^{32}$ & \multirow{2}{*}{$\begin{array}{l}\text { Comparar a eficácia entre } \\
\text { açafrão e triancinolona no } \\
\text { tratamento de úlcera aftosa } \\
\text { recorrente menor na cavida- } \\
\text { de oral (uso tópico). }\end{array}$} & \multirow{2}{*}{$\begin{array}{l}\text { Os dois tratamentos apresentaram uma } \\
\text { eficácia similar, pois aliviaram a dor e } \\
\text { reduziram o tamanho das úlceras aftosas } \\
\text { recorrentes. } \\
\text { Não foram encontradas diferenças signi- } \\
\text { ficativas entre os dois grupos estudados. } \\
\text { Considerando que a triancinolona não } \\
\text { pode ser utilizada no manejo de longo } \\
\text { prazo no tratamento da úlcera, como } \\
\text { alternativa, o açafrão pode ser utilizado } \\
\text { para o tratamento da úlcera aftosa recor- } \\
\text { rente menor. }\end{array}$} \\
\hline 2013 & $\begin{array}{l}\text { Ensaio clínico rando- } \\
\text { mizado cego }\end{array}$ & & \\
\hline $\begin{array}{l}\text { A clinical evaluation of } \\
5 \% \text { amlexanox oral paste } \\
\text { in the treatment of minor } \\
\text { recurrent aphthous ulcers and } \\
\text { comparison with the placebo } \\
\text { paste: A randomized, vehicle } \\
\text { controlled, parallel, single } \\
\text { center clinical trial }\end{array}$ & Bhat e Sujatha ${ }^{33}$ & \multirow[t]{2}{*}{$\begin{array}{l}\text { Comparar a eficácia da pas- } \\
\text { ta oral com amlexanox } 5 \% \\
\text { (uso tópico) no tratamento } \\
\text { de úlceras aftosas recorren- } \\
\text { tes menores bem como ava- } \\
\text { liar a taxa de recorrência de } \\
\text { úlceras aftosas durante um } \\
\text { período de um ano. }\end{array}$} & \multirow[t]{2}{*}{$\begin{array}{l}\text { O tratamento com a pasta de amlexa- } \\
\text { nox mostrou uma redução acentuada no } \\
\text { tamanho da úlcera ( } p<0,001) \text {, redução } \\
\text { de dor }(p<0,001) \text {, e reduções significa- } \\
\text { tivas de eritema e exsudação }(p<0,001) \\
\text { quando comparados com o tratamento } \\
\text { placebo no sexto dia de acompanha- } \\
\text { mento. Houve redução da incidência da } \\
\text { recorrência de úlceras até o sexto mês, } \\
\text { no entanto, após esse período, a inci- } \\
\text { dência aumentou novamente. }\end{array}$} \\
\hline 2013 & $\begin{array}{l}\text { Ensaio clínico rando- } \\
\text { mizado }\end{array}$ & & \\
\hline $\begin{array}{l}\text { Levamisole and low-dose } \\
\text { prednisolone in the treatment } \\
\text { of reccurentaphthous } \\
\text { stomatitis }\end{array}$ & Sharda et al. ${ }^{34}$ & \multirow{2}{*}{$\begin{array}{l}\text { Comparar a resposta de pa- } \\
\text { cientes com estomatite afto- } \\
\text { sa recorrente ao tratamento } \\
\text { (sistêmico) com levamisol }+ \\
\text { baixa dose de prednisolona, } \\
\text { levamisol e placebo. }\end{array}$} & \multirow{2}{*}{$\begin{array}{l}\text { Os resultados mostraram que a admi- } \\
\text { nistração do levamisol sozinho e da } \\
\text { combinação de levamisol + predniso- } \\
\text { lona tiveram a mesma performance em } \\
\text { alterar o curso da doença, no entanto, } \\
\text { a administração do levamisol + predni- } \\
\text { solona foi sutilmente melhor em reduzir } \\
\text { a severidade da doença, dessa forma, a } \\
\text { combinação de levamisol + prednisolo- } \\
\text { na mostrou-se mais eficaz na redução de } \\
\text { número de úlceras/episódios e redução } \\
\text { da dor. }\end{array}$} \\
\hline 2014 & $\begin{array}{l}\text { Ensaio clínico rando- } \\
\text { mizado cego controla- } \\
\text { do por placebo }\end{array}$ & & \\
\hline $\begin{array}{l}\text { A randomized, open label, } \\
\text { clinical study of synbiotics in } \\
\text { patients with recurrent minor } \\
\text { aphthous ulcers }\end{array}$ & Aswath et al. ${ }^{35}$ & \multirow{2}{*}{$\begin{array}{l}\text { Avaliar a eficácia e rapidez } \\
\text { do efeito para um simbióti- } \\
\text { co em pastilha (uso sistêmi- } \\
\text { co) no tratamento da EAR. }\end{array}$} & \multirow{2}{*}{$\begin{array}{l}\text { O estudo demonstrou rapidez e eficácia } \\
\text { das respostas das pastilhas de simbióti- } \\
\text { cos em solucionar a EAR. Primeiro es- } \\
\text { tudo a confirmar a eficácia das pastilhas } \\
\text { de simbióticos para resolução da EAR }\end{array}$} \\
\hline 2014 & $\begin{array}{l}\text { Ensaio clínico rando- } \\
\text { mizado }\end{array}$ & & \\
\hline $\begin{array}{l}\text { Efficacy of omega-3 in } \\
\text { treatment of recurrent } \\
\text { aphthous stomatitis and } \\
\text { improvement of quality of } \\
\text { life: a randomized, double- } \\
\text { blind, placebo-controlled } \\
\text { study }\end{array}$ & El Khouli e El-Gendy ${ }^{36}$ & \multirow{2}{*}{$\begin{array}{l}\text { Avaliar o potencial da su- } \\
\text { plementação alimentar de } \\
\text { ácidos graxos poli-insatura- } \\
\text { dos ômega } 3 \mathrm{em} \text { promover } \\
\text { uma terapêutica acessível, } \\
\text { segura e efetiva para o tra- } \\
\text { tamento da estomatite aftosa } \\
\text { recorrente. }\end{array}$} & \multirow{2}{*}{$\begin{array}{l}\text { Os resultados mostraram que, após três } \\
\text { meses de tratamento com os ácidos gra- } \\
\text { xos poli-insaturados ômega } 3 \text {, houve } \\
\text { diferenças significativas na média de } \\
\text { surtos mensais de lesões aftosas, esco- } \\
\text { re da escala visual de dor e média de } \\
\text { duração dos episódios de úlcera quando } \\
\text { se comparou o grupo teste com o grupo } \\
\text { controle. }\end{array}$} \\
\hline 2014 & $\begin{array}{l}\text { Ensaio clínico randomi- } \\
\text { zado duplo-cego con- } \\
\text { trolado por placebo }\end{array}$ & & \\
\hline
\end{tabular}


Quadro 3 - Síntese dos estudos incluídos na amostra que utilizaram laser para o tratamento da EAR, segundo título, ano, autoria, tipo de estudo, objetivos, resultados e conclusões

\begin{tabular}{|c|c|c|c|}
\hline Título & Autoria & \multirow{2}{*}{ Objetivos } & \multirow{2}{*}{ Resultados/conclusões } \\
\hline Ano & Tipo de estudo & & \\
\hline $\begin{array}{l}\text { Assessment of immediate } \\
\text { pain relief with laser } \\
\text { treatment in recurrent } \\
\text { aphthous stomatitis }\end{array}$ & Prasad e Pai ${ }^{19}$ & \multirow{2}{*}{$\begin{array}{l}\text { Avaliar a eficácia de uma } \\
\text { única sessão de tratamen- } \\
\text { to com laser de } \mathrm{CO}_{2} \text { com } \\
\text { comprimento de onda de } \\
10.6 \mathrm{~nm} \text {, em modo não } \\
\text { ablativo, em promover o } \\
\text { alívio imediato da dor de } \\
\text { pacientes com ulceração af- } \\
\text { tosa recorrente menor. }\end{array}$} & \multirow{2}{*}{$\begin{array}{l}\text { Uma única irradiação com laser de } \mathrm{CO}_{2} \\
\text { em lesões de úlcera aftosa recorrente me- } \\
\text { nor promoveu um alívio imediato signifi- } \\
\text { cativo da dor. O tratamento também mos- } \\
\text { trou um efeito positivo no tempo de ci- } \\
\text { catrização. Mais estudos são necessários } \\
\text { para confirmar os mecanismos envolvidos } \\
\text { na produção de analgesia e aceleração do } \\
\text { processo de cicatrização com a utilização } \\
\text { do laser de } \mathrm{CO}_{2} \text {. }\end{array}$} \\
\hline 2013 & $\begin{array}{l}\text { Ensaio clínico } \\
\text { controlado cruzado }\end{array}$ & & \\
\hline $\begin{array}{l}\text { Efficacy of low-level laser } \\
\text { therapy in treatment of } \\
\text { recurrent aphthous ulcers } \\
\text { - a sham controlled, split } \\
\text { mouth follow up study }\end{array}$ & Aggarwal et al. ${ }^{37}$ & \multirow{2}{*}{$\begin{array}{l}\text { Avaliar clinicamente a efi- } \\
\text { cácia da terapia com laser } \\
\text { de baixa potência sobre a } \\
\text { EAR para redução da dor, } \\
\text { tamanho da lesão e tempo } \\
\text { de cicatrização, comparan- } \\
\text { do com um grupo controle } \\
\text { (placebo). }\end{array}$} & \multirow{2}{*}{$\begin{array}{l}\text { Resolução completa da lesão em menor } \\
\text { tempo após aplicação do laser, quando } \\
\text { comparado com o grupo controle. Com } \\
\text { relação à dor, no grupo teste, após uma } \\
\text { sessão, } 28 \text { pacientes não sentiam dor, e } \\
\text { após dois dias, trinta pacientes. A terapia } \\
\text { com laser de baixa potência é eficaz na } \\
\text { redução da dor e do tempo de cicatri- } \\
\text { zação na EAR. }\end{array}$} \\
\hline 2014 & $\begin{array}{l}\text { Ensaio clínico de } \\
\text { segmento controlado, } \\
\text { boca dividida }\end{array}$ & & \\
\hline $\begin{array}{l}\text { Recurrent aphthous } \\
\text { stomatitis and pain } \\
\text { management with low-level } \\
\text { laser therapy: a randomized } \\
\text { controlled trial }\end{array}$ & Albrektson et al. ${ }^{38}$ & \multirow{2}{*}{$\begin{array}{l}\text { Determinar se o laser de } \\
\text { baixa intensidade tem efei- } \\
\text { to analgésico em pacientes } \\
\text { com EAR. }\end{array}$} & \multirow{2}{*}{$\begin{array}{l}\text { O tratamento com laser de baixa potência } \\
\text { aliviou a dor decorrente da EAR nesse es- } \\
\text { tudo quando comparado ao grupo place- } \\
\text { bo. Também reduziu a dor dos pacientes } \\
\text { ao comer, beber e escovar os dentes. }\end{array}$} \\
\hline 2014 & $\begin{array}{l}\text { Ensaio clínico } \\
\text { randomizado } \\
\text { controlado }\end{array}$ & & \\
\hline
\end{tabular}

\section{Discussão}

Nos últimos anos, a indústria farmacêutica vem investindo no desenvolvimento de medicamentos originados de produtos naturais com o objetivo de superar a resistência a alguns tipos de drogas, como antimicrobianos, e na busca de uma melhor relação custo/efetividade em relação às substâncias sintéti$\operatorname{cas}^{16}$. Nesse contexto, dos 23 estudos incluídos nesta revisão integrativa, sete artigos avaliaram o uso tópico de medicamentos naturais no manejo da EAR (Quadro 1).

Diferentes substâncias naturais foram pesquisadas, e a maioria dos estudos encontrou resultados significativos na redução da dor ${ }^{16,21-27}$, redução do tamanho da lesão ${ }^{16,21-27}$, menor tempo de duração da úlcera $^{21,23,26,27}$, eritema e exsudado ${ }^{23,25,27}$, sem relatos, portanto, de efeitos colaterais decorrentes da utilização das substâncias naturais estudadas.

Desse modo, a eficácia dessas substâncias pode ser atribuída às propriedades que auxiliam o manejo da EAR, como os efeitos analgésicos, anti-inflamatórios, antimicrobianos, antissépticos, cicatrizantes, antioxidantes e imunomoduladores.

Além dos medicamentos naturais, os estudos presentes nesta revisão integrativa incluíram também treze estudos com substâncias farmacológicas convencionais com aplicação tópica ou com uso sistêmico (Quadro 2), como antibióticos, anti-inflamatórios, vitaminas, ácidos graxos e outras classes medicamentosas. Dois ensaios clínicos presentes nesta revisão integrativa estudaram a utilização de antibióticos no manejo da EAR, o hiclato de doxiciclina ${ }^{5}$, em aplicação tópica única, e a penicilina $\mathrm{G}$ potássica ${ }^{28}$, em aplicação tópica quatro vezes ao dia. A doxiciclina mostrou-se eficaz na redução da dor causada pela úlcera, promovendo recuperação acelerada, e a penicilina $\mathrm{G}$ potássica também atuou no alívio da dor e auxiliou, ainda, na redução do tamanho da úlcera.

Regimes tópicos e sistêmicos de tetraciclinas têm sido utilizados no manejo da EAR, não somente devido às suas propriedades antimicrobianas, mas também devido a outras propriedades, tais como inibição da produção de prostaglandinas, supressão de leucócitos, inibição de colagenases e gelatinases ${ }^{28}$. Quanto à utilização de penicilinas, embora o mecanismo de ação na EAR permaneça indefinido, acredita-se que a eficácia esteja relacionada aos efeitos do antibiótico contra o Streptococcus $\mathrm{spp}^{5}$.

Substâncias anti-inflamatórias esteroides e não esteroides, como a pomada de dexametasona (uso tópico) ${ }^{17}$, a pasta de amlexanox (uso tópico) ${ }^{33}$ e a combinação de levamisol e prednisolona (uso sistêmico ${ }^{34}$, também foram utilizadas no tratamento da estomatite aftosa recorrente.

Todos esses estudos consistiram em ensaios clínicos com satisfatórios efeitos para o tratamento da condição. A pomada de dexametasona foi testada quanto à aplicação tópica três vezes ao dia, apresentando resultados satisfatórios na redução do tamanho e tempo de duração das úlceras e aceleração da 
cicatrização. A pasta de amlexanox, aplicada quatro vezes ao dia, foi eficaz na redução do tamanho da úlcera, alívio da dor, redução do eritema e exsudação. O levamisol, um imunopotenciador, também foi eficaz no tratamento do EAR quando administrado sozinho (comprimidos de $50 \mathrm{mg}$ ) e quando administrado em combinação com a prednisolona $(50 \mathrm{mg}$ de levamisol e $5 \mathrm{mg}$ de prednisolona), com resultados mais promissores no último caso.

Dois estudos compararam os efeitos terapêuticos de produtos naturais com os efeitos de fármacos convencionais. Quando se comparou os efeitos terapêuticos da prednisolona com os da colchicina no tratamento da EAR (uso sistêmico), as duas terapias tiveram uma eficácia semelhante, no entanto, a colchicina ( $5 \mathrm{mg}$ ) foi associada com efeitos colaterais mais frequentes, dessa forma, a prednisolona parece ser uma alternativa melhor na redução dos sinais e sintomas da doença ${ }^{29}$.

Em contrapartida, quando se comparou os efeitos do uso do açafrão com a triancinolona (uso tópico), apesar dos dois tratamentos apresentarem eficácia semelhante, recomendou-se o uso do açafrão, considerando que a triancinolona não pode ser utilizada por longos períodos devido ao fato de ser um anti-inflamatório esteroide ${ }^{32}$.

A presente revisão agregou ainda outras substâncias e terapias, como a administração sistêmica de ascorbato, que se mostrou eficaz no manejo da EAR com diminuição da frequência de aparecimento de lesões e diminuição da severidade de episódios, contudo, os autores concluíram que os resultados são preliminares, havendo a necessidade de novos estudos ${ }^{18}$; a suplementação sistêmica polivitamínica, que não mostrou resultados satisfatórios quanto à diminuição de novos episódios de úlceras ${ }^{31}$; a terapêutica sistêmica com ômega 3 , que resultou em diminuição dos surtos mensais de EAR após terapia por três meses, além de diminuir a dor e a média de duração dos episódios ${ }^{36}$; a aplicação tópica de diosmectite combinado com pasta de fator de crescimento fibroblástico, que se mostrou eficaz na diminuição da dor e promoção da cicatrização da mucosa bucal ${ }^{16}$; a administração tópica de pastilhas de simbióticos (pacientes instruídos a mastigar a pastilha), que também se mostrou eficaz quanto à diminuição do número de úlceras por episódios e redução do tamanho das úlceras ${ }^{35}$.

Outra modalidade de tratamento, apresentada por três artigos, foi o uso de laser de baixa potência (Quadro 3), considerada uma alternativa de tratamento por oferecer melhor resposta inflamatória com redução de edema e de dor, além da bioestimulação celular para regeneração tecidual ${ }^{39}$. Prasad e $\mathrm{Pai}^{19}$ analisaram o efeito da aplicação de uma única sessão de laser de $\mathrm{CO}_{2}$ no alívio da dor provocada pela úlcera, e concluíram que esse tratamento foi eficaz, além de diminuir o tempo de cicatrização da úlcera. Resultados semelhantes foram encontrados no estudo conduzido por Aggarwal et al. ${ }^{37}$, com me- lhor tempo de cicatrização completa das úlceras e com alívio da dor imediata. Albrektson et al. ${ }^{38}$ também avaliaram a eficácia do laser de baixa potência no alívio da dor característica dos episódios de EAR com a apresentação de resultados analgésicos em relação ao tratamento placebo e relatos de alívio da dor em atividades do cotidiano, como ao comer, beber ou escovar os dentes, alcançando o valor zero na escala visual analógica (VAS) no terceiro dia da aplicação.

A maioria dos estudos foi realizada em pacientes adultos, sendo que apenas um foi realizado em adolescentes. O tipo de úlcera mais frequentemente tratado foi a estomatite aftosa recorrente menor de causa idiopática. Dos 23 estudos analisados, 22 são classificados como ensaios clínicos e um como revisão sistemática ${ }^{30}$.

Os estudos analisados apresentaram alguns vieses que podem ter influenciado os resultados, dentre eles, a falta de cálculo amostral, a ausência de grupo controle, a falta de randomização ou não explicitação dos critérios de randomização, as perdas amostrais não especificadas e a falta de cegamento ou cegamento incompleto. Dessa forma, verifica-se a necessidade da realização de estudos mais robustos com as sustâncias testadas para a obtenção de um nível de evidência científica mais consistente.

\section{Considerações finais}

Uma grande variedade de substâncias e/ou terapias tem sido estudada como tratamento da estomatite aftosa recorrente, havendo, muitas vezes, a apresentação de satisfatórios resultados quanto ao fármaco ou à terapêutica utilizados.

O presente estudo sugere cautela quanto à indicação das terapias no manejo da EAR, considerando as terapias tópicas como um todo, desde os medicamentos naturais até a terapia com laser, opções eficazes de tratamento e com menores possibilidades de efeitos colaterais.

Quanto aos tratamentos sistêmicos, este estudo considerou pertinente o mencionado em uma revisão sistemática previamente publicada, que atesta que os resultados permanecem inconclusivos no que diz respeito às melhores intervenções sistêmicas para a estomatite aftosa recorrente.

\section{Abstract}

Objective: To perform an integrative review of scientific publications on the treatment of recurrent aphthous stomatitis, seeking resources to guide the clinical practice in the management of this condition. Literature review: The recurrent aphthous stomatitis is characterized by the appearance of ulcers in the oral mucosa, which are presented as painful lesions and lead to a negative impact on the quality of life of patients. This study performed a search on recurrent aphthous stomatitis 
in the following databases: PubMed (National Center for Biotechnology Information and National Library of Medicine), The Cochrane Library, and SCOPUS (The Scopus Cited Reference Expansion), using the following keywords: recurring aphthous stomatitis $O R$ recurrent aphthous stomatitis $O R$ recurrent aphthous ulcer $O R$ recurrent aphthous ulceration AND treatment. Twenty-three studies were selected according to inclusion and exclusion criteria applied. These studies identified the use of several drugs, ranging from herbal remedies to laser for both topical and systemic use, with satisfactory results for the management of recurrent aphthous stomatitis. Final considerations: This study considered topical treatments, as a whole, an effective treatment option with less potential for side effects. Regarding the systemic therapies, the results remain inconclusive.

Keywords: Aphthous stomatitis. Treatment. Pathology.

\section{Referências}

1. Jurge S, Kuffer R, Scully C, Porter SR. Mucosal disease series. Number VI. Recurrent aphthous stomatitis. Oral Dis 2006;12(1):1-21.

2. Shulman JD. An exploration of point, annual, and lifetime prevalence in characterizing recurrent aphthous stomatitis in USA children and youths. J Oral Pathol Med 2004; 33(9):558-66.

3. Akintoye SO, Greenberg MS. Recurrent aphthous stomatitis. Dent Clin North Am 2005; 49(1):31-47, vii-viii.

4. Besu I, Jankovic L, Magdu IU, Konic-Ristic A, Raskovic S, Juranic Z. Humoral immunity to cow's milk proteins and gliadin within the etiology of recurrent aphthous ulcers? Oral Dis 2009; 15(8):560-4.

5. Vijayabala GS, Kalappanavar AN, Annigeri RG, Sudarshan R, Shettar SS. Single application of topical doxycycline hyclate in the management of recurrent aphthous stomatitis. Oral Surg Oral Med Oral Pathol Oral Radiol 2013; 116(4):440-6.

6. Mills MP, Mackler BF, Nelms DC, Peavy DL. Quantitative distribution of inflammatory cells in recurrent aphthous stomatitis. J Dent Res 1980; 59(3):562-6.

7. Wray D, Charon J. Polymorphonuclear neutrophil function in recurrent aphthous stomatitis. J Oral Pathol Med 1991; 20(8):392-4.

8. Sistig S, Cekic-Arambasin A, Rabatic S, Vucicevic-Boras V, Kleinheinz J, Piffko J. Natural immunity in recurrent aphthous ulceration. J Oral Pathol Med 2001; 30(5):275-80.

9. Natah SS, Hayrinen-Immonen R, Hietanen J, Malmstrom $\mathrm{M}$, Konttinen YT. Immunolocalization of tumor necrosis factor-alpha expressing cells in recurrent aphthous ulcer lesions (RAU). J Oral Pathol Med 2000; 29(1):19-25.

10. Belenguer-Guallar I, Jimenez-Soriano Y, Claramunt-Lozano A. Treatment of recurrent aphthous stomatitis. A literature review. J Clin Exp Dent 2014; 6(2):e168-74.

11. Tabolli S, Bergamo F, Alessandroni L, Di Pietro C, Sampogna F, Abeni D. Quality of life and psychological problems of patients with oral mucosal disease in dermatological practice. Dermatology 2009; 218(4):314-20.

12. Ship JA, Chavez EM, Doerr PA, Henson BS, Sarmadi M. Recurrent aphthous stomatitis. Quintessence Int 2000; 31(2):95-112

13. Huling LB, Baccaglini L, Choquette L, Feinn RS, Lalla RV. Effect of stressful life events on the onset and duration of recurrent aphthous stomatitis. J Oral Pathol Med 2012; 41(2):149-52

14. Barrons RW. Treatment strategies for recurrent oral aphthous ulcers. Am J Health Syst Pharm 2001; 58(1):41-50; quiz 51-3.

15. Greenberg MS, Pinto A. Etiology and management of recurrent aphthous stomatitis. Curr Infect Dis Rep 2003; 5(3):194-8.

16. Jiang XW, Zhang Y, Zhang H, Lu K, Yang SK, Sun GL. Double-blind, randomized, controlled clinical trial of the effects of diosmectite and basic fibroblast growth factor paste on the treatment of minor recurrent aphthous stomatitis. Oral Surg Oral Med Oral Pathol Oral Radiol 2013; 116(5):570-5.

17. Liu C, Zhou Z, Liu G, Wang Q, Chen J, Wang L et al. Efficacy and safety of dexamethasone ointment on recurrent aphthous ulceration. Am J Med 2012; 125(3):292-301.

18. Yasui K, Kurata T, Yashiro M, Tsuge M, Ohtsuki S, Morishima T. The effect of ascorbate on minor recurrent aphthous stomatitis. Acta Paediatr 2010; 99(3):442-5.

19. Prasad R, Pai A. Assessment of immediate pain relief with laser treatment in recurrent aphthous stomatitis. Oral Surg Oral Med Oral Pathol Oral Radiol 2013; 116(2):189-93.

20. McGrath JM. Systematic and integrative reviews of the literature: how are they changing our thoughts about practice? J Perinat Neonatal Nurs 2012; 26(3):193-5.

21. Pourahmad M, Rahiminejad M, Fadaei S, Kashafi H. Effects of camel thorn distillate on recurrent oral aphthous lesions. J Dtsch Dermatol Ges 2010; 8(5):348-52.

22. Hamdy AA, Ibrahem MA. Management of aphthous ulceration with topical quercetin: a randomized clinical trial. J Contemp Dent Pract 2010; 11(4):E009-16.

23. Babaee N, Mansourian A, Momen-Heravi F, Moghadamnia A, Momen-Beitollahi J. The efficacy of a paste containing Myrtus communis (Myrtle) in the management of recurrent aphthous stomatitis: a randomized controlled trial. Clin Oral Investig 2010; 14(1):65-70.

24. Jiang XW, Zhang Y, Song GD, Li FF, Peng HY, Yang SK et al. Clinical evaluation of allicin oral adhesive tablets in the treatment of recurrent aphthous ulceration. Oral Surg Oral Med Oral Pathol Oral Radiol 2012 Apr; 113(4):500-4.

25. Jiang XW, Zhang Y, Zhu YL, Zhang H, Lu K, Li FF et al. Effects of berberine gelatin on recurrent aphthous stomatitis: a randomized, placebo-controlled, double-blind trial in a Chinese cohort. Oral Surg Oral Med Oral Pathol Oral Radiol 2013; 115(2):212-7.

26. He Y, Gong D, Zhu R. The efficacy of Kasmitad gel in the management of recurrent minor aphthous ulceration. Kuwait Med J 2014; 46(2):124-9.

27. Bechir A, Sirbu R, Pacurar M, Podariu AC, Monea M, Bechir ES et al. The effect of collagenic gels with marine algae extracts mixtures in the treatment of recurrent aphthous stomatitis. Revista de Chimie 2014; 362-8.

28. Zhou Y, Chen Q, Meng W, Jiang L, Wang Z, Liu J et al. Evaluation of penicillin $\mathrm{G}$ potassium troches in the treatment of minor recurrent aphthous ulceration in a Chinese cohort: a randomized, double-blinded, placebo and no-treatment-controlled, multicenter clinical trial. Oral Surg Oral Med Oral Pathol Oral Radiol Endod 2010; 109(4):561-6.

29. Pakfetrat A, Mansourian A, Momen-Heravi F, Delavarian Z, Momen-Beitollahi J, Khalilzadeh O et al. Comparison of colchicine versus prednisolone in recurrent aphthous stomatitis: a double-blind randomized clinical trial. Clin Invest Med 2010; 33(3):E189-95.

30. Brocklehurst P, Tickle M, Glenny AM, Lewis MA, Pemberton MN, Taylor $\mathrm{J}$ et al. Systemic interventions for recurrent 
aphthous stomatitis (mouth ulcers). Cochrane Database Syst Rev 2012; 9:CD005411.

31. Lalla RV, Choquette LE, Feinn RS, Zawistowski H, Latortue MC, Kelly ET et al. Multivitamin therapy for recurrent aphthous stomatitis: a randomized, double-masked, placebo-controlled trial. J Am Dent Assoc 2012; 143(4):370-6.

32. Halim DS, Abdullah NA, Alam MK, Samsee SNB, May TS. Comparasion of the effectiveness between virgin coconut oil (VCO) and triamcinolone for treatment of minor recurrent aphthous stomatitis (RAS). Int Med J 2014; 21(3):319-20.

33. Bhat S, Sujatha D. A clinical evaluation of 5\% amlexanox oral paste in the treatment of minor recurrent aphthous ulcers and comparison with the placebo paste: a randomized, vehicle controlled, parallel, single center clinical trial. Indian J Dent Res 2013; 24(5):593-8.

34. Sharda N, Shashikanth MC, Kant P, Jain M. Levamisole and low-dose prednisolone in the treatment of reccurent aphthous stomatitis. J Oral Pathol Med 2014; 43(4):309-16.

35. Aswath N, Praveen Kumar ST, Raghavendra Jayesh S, Manigandan T, Sarumathi T. A randomized, open label, clinical study of synbiotics in patients with recurrent minor aphthous ulcers. Res J Pharm Biol Chem Sci 2014; 5(2):1900-5.

36. El Khouli AM, El-Gendy EA. Efficacy of omega-3 in treatment of recurrent aphthous stomatitis and improvement of quality of life: A randomized, double-blind, placebo-controlled study. Oral Surg Oral Med Oral Pathol Oral Radiol 2014; 117(2):191-6

37. Aggarwal H, Singh MP, Nahar P, Mathur H, Gv S. Efficacy of low-level laser therapy in treatment of recurrent aphthous ulcers - a sham controlled, split mouth follow up study. J Clin Diagn Res 2014; 8(2):218-21.

38. Albrektson M, Hëadstrom L, Bergh H. Recurrent aphthous stomatitis and pain management with low-level laser therapy: a randomized controlled trial. Oral Surg Oral Med Oral Pathol Oral Radiol 2014; 117(5):590-4.

39. Yarak S, Okamoto OK. Biostimulation effects of low power laser in the repair process. An Bras Dermatol 2010; 85(6):849-55.

\section{Endereço para correspondência:}

Anna Clara Fontes Vieira

Campus Universitário do Bacanga

Av. dos Portugueses, 1.996

65080-805 São Luís, MA

Telefone: (098) 8813-9386

E-mail: clara_afv@hotmail.com

Recebido: 15/12/14. Aceito: 15/05/15. 